\title{
Transients of platoons with asymmetric and different Laplacians ${ }^{\underline{k}}$
}

\author{
Ivo Herman ${ }^{\mathrm{a}, *}$, Dan Martinec ${ }^{\mathrm{a}}$, J. J. P. Veerman ${ }^{\mathrm{b}, \mathrm{c}}$ \\ ${ }^{a}$ Faculty of Electrical Engineering, Czech Technical University in Prague. Czech Republic \\ ${ }^{b}$ Fariborz Maseeh Dept. of Math. and Stat., Portland State Univ., Portland, OR, USA \\ ${ }^{c}$ CCQCN, Dept of Physics, University of Crete, 71003 Heraklion, Greece
}

\begin{abstract}
We consider an asymmetric control of platoons of identical vehicles with nearest-neighbor interaction. Recent results show that if the vehicle uses different asymmetries for position and velocity errors, the platoon has a short transient and

n low overshoots. In this paper we investigate the properties of vehicles with friction. To achieve consensus, an integral part is added to the controller, making the vehicle a third-order system. We show that the parameters can be chosen so that the platoon behaves as a wave equation with different wave velocities. Simulations suggest that our system has a better performance than other nearest-neighbor scenarios. Moreover, an optimization-based procedure is used to find the controller properties.

Keywords: vehicular platoons, different asymmetries, distributed control, scaling, multiple Laplacian
\end{abstract}

ก

N

\section{Introduction}

Control of vehicular platoons has become a field of intensive research. The reason for this is the possibility to increase throughput and safety of the highway traffic at the same time. Moreover, since the study of such large systems gives asymptotic behavior and achievable limits, $\checkmark$ it is also appealing from a theoretical perspective.

It is well known that if some centralized information is present in the system, then the performance is good and the system is scalable. These approaches comprise either LQR control [1] or local control with added knowledge of the desired velocity [2, 3]. Among the systems with permanent communication the Cooperative Automatic Cruise Control is the most widely studied 4 and implemented.

In this paper we consider a nearest-neighbor interaction without any centralized information. The most important performance measure is, apart from the settling time, a so called string stability. The system is string stable if the disturbance acting at one vehicle does not amplify as it propagates along the platoon [5]. It is shown in [6] that a time-headway spacing policy can achieve string stability. However, this policy increases the platoon length with the speed of the platoon.

J.J.P. Veerman's research was partially supported by the European Union's Seventh Framework Program (FP7-REGPOT-20122013-1) under grant agreement n316165. I. Herman was supported by the Czech Science Foundation within the project GACR 13-06894S. For the simulations we used the code written by Carlos Cantos.

* Corresponding author

Email addresses: ivo.herman@fel.cvut.cz (Ivo Herman), dan.martinec@fel.cvut.cz (Dan Martinec), veerman@pdx.edu (J. J. P. Veerman)
A common fixed-distance scenario is a symmetric bidirectional control, where the vehicle measures the distance to both its neighbors in the platoon. The properties of such control are investigated in [2, 7, 8]. One of the advantages is linear scaling of the $\mathcal{H}_{\infty}$ norm with the number of vehicles 8 . The main drawback is a very long transient for a higher number of vehicles and sensitivity to noise [9]. Both can be qualitatively decreased using a feedback control of the leader [10].

Asymmetric control was proposed to shorten the transient and increase the controllability of large platoons [3. 11 - a vehicle uses different weights to its two neighbors. Nevertheless, the price to pay is an exponential growth of $\mathcal{H}_{\infty}$ norm of the transfer functions with the graph distance between vehicles 12, 13, so this approach is not scalable.

Both symmetric and asymmetric control share one common property - the asymmetry in coupling between between vehicles is the same for position and velocity. That is, only one graph Laplacian is used. Except for a simpler analysis, there are not many reasons to limit ourselves to use only one type of coupling. As was numerically shown in [14] and more thoroughly discussed in 15, the response of a platoon with a symmetric coupling in position and asymmetric in velocity can be scalable and have short transients. Thus, this approach combines the advantages both from symmetric and asymmetric control.

The paper 15] considers a double integrator model of the system and uses wave properties for system analysis. In this paper we extend the results of [15] to a third-order system. The main reason is the presence of friction in every real vehicle. We added an integrator to the system to achieve coherent solutions. With such a system, the stability and performance analysis of the platoon complicates. 
Although a similar type of a system was recently analyzed in [16, the results are not applicable for our case since the coupling used in our work is non-symmetric.

Finally, to achieve a good performance, we provide an optimization procedure based on the properties of waves in the platoon. This approach allows us to optimize the controller parameters and weights of the communication graph at the same time.

\section{Model of the vehicles}

We assume $N+1$ identical vehicles travelling on a line, indexed as $0, \ldots, N$. The first vehicle with index 0 is a leader which is driven independently of the the rest of the formation. Unlike standard double integrator models [9, 12, 15, real systems have a friction, i. e., there is a feedback from velocity, which eventually makes the vehicle to stop. The vehicle model is

$$
\ddot{x}_{i}=-a v_{i}+u_{i},
$$

where $x_{i}$ is the position of the $i$ th vehicle, $v_{i}=\dot{x}_{i}$ is its velocity, $a \in \mathbb{R}$ is the viscous friction coefficient and $u_{i}$ is the input to the vehicle.

In order to enable the vehicles in the platoon to track the leader moving with constant velocity, we need to satisfy the Internal Model Principle [17, 18 which in our case means the presence of two integrators in the openloop model of each vehicle. Since one integrator is already present in the vehicle model $\left(\dot{x}_{i}=v_{i}\right)$, it suffices to add an integral action in the controller of each vehicle. The controller is given as $\dot{\bar{c}}_{i}=\epsilon_{i}$ with $\epsilon_{i}$ defined in (2) and $\bar{c}_{i}$ is the state of the integrator in the controller. The input to the vehicle is then $u_{i}=\bar{c}_{i}$.

Each vehicle uses only the information obtained from its nearest neighbors - the vehicle in front of it and behind. The goal of the vehicle is to keep a prescribed spacing to them, i.e., $x_{i-1}-x_{i} \rightarrow d_{i-1, i}$ with $d_{i, j}$ being the desired distance between $i$ and $j$. The regulation error $\epsilon_{i}$ comes from the relative spacing and velocity errors as

$$
\begin{aligned}
\epsilon_{i}= & g_{x}\left[\left(1-\rho_{x}\right)\left(x_{i-1}-x_{i}-d_{i-1, i}\right)-\rho_{x}\left(x_{i}-x_{i+1}\right.\right. \\
& \left.\left.-d_{i, i+1}\right)\right]+g_{v}\left[\left(1-\rho_{v}\right)\left(v_{i-1}-v_{i}\right)-\rho_{v}\left(v_{i}-v_{i+1}\right)\right],
\end{aligned}
$$

where the position asymmetry is labeled as $\rho_{x}$, velocity asymmetry as $\rho_{v}$ and the $g_{x}, g_{v} \in \mathbb{R}$ are weights of position and velocity errors. The coupling in position is symmetric if $\rho_{x}=0.5$ and asymmetric otherwise (the same for $\rho_{v}$ ).

To simplify the analysis, we introduce error variables $z_{i}=x_{i}-x_{0}+d_{0, i}$. This implies $z_{i-1}-z_{i}=x_{i-1}-x_{i}-d_{i-1, i}$ and $\dot{z}_{i-1}-\dot{z}_{i}=\dot{x}_{i-1}-\dot{x}_{i}$. We impose that $d_{i, k}-d_{i, j}=d_{j, k}$ and $d_{i, j}=-d_{j, i}$. The single vehicle model combined with the controller then has a form

$$
\ddot{z}_{i}=-a \dot{z}_{i}+\bar{c}, \quad \dot{\overline{c_{i}}}=\epsilon_{i} .
$$

We use a minor state transformation $c_{i}=\bar{c}_{i}-a v_{i}$ to obtain a controller-canonical form of the individual-vehicle model

$$
\left(\begin{array}{c}
\dot{z}_{i} \\
\ddot{z}_{i} \\
\dot{c}_{i}
\end{array}\right)=\left(\begin{array}{c}
\dot{z}_{i} \\
\ddot{z}_{i} \\
\ddot{z}_{i}
\end{array}\right)=\left(\begin{array}{ccc}
0 & 1 & 0 \\
0 & 0 & 1 \\
0 & 0 & -a
\end{array}\right)\left(\begin{array}{c}
z_{i} \\
\dot{z}_{i} \\
\ddot{z}_{i}
\end{array}\right)+\left(\begin{array}{l}
0 \\
0 \\
1
\end{array}\right) \epsilon_{i} .
$$

In a vector form we write the overall system of $N+1$ vehicles (including the leader) as

$$
\frac{d}{d t}\left(\begin{array}{c}
z \\
\dot{z} \\
\ddot{z}
\end{array}\right)=M_{N}\left(\begin{array}{l}
z \\
\dot{z} \\
\ddot{z}
\end{array}\right) \equiv\left(\begin{array}{ccc}
0 & I & 0 \\
0 & 0 & I \\
-g_{x} L_{x} & -g_{v} L_{v} & -a I
\end{array}\right)\left(\begin{array}{l}
z \\
\dot{z} \\
\ddot{z}
\end{array}\right),
$$

where $z=\left[z_{0}, \ldots, z_{N}\right]^{T}$. Let us call the system (5) a path system, since the communication topology is a weighted path graph. The Laplacians $L_{x}, L_{v} \in \mathbb{R}^{N+1 \times N+1}$ of the path graph are defined as

$$
\begin{aligned}
& L_{x}=\left(\begin{array}{cccccc}
0 & 0 & 0 & 0 & \ldots & 0 \\
-\left(1-\rho_{x}\right) & 1 & -\rho_{x} & 0 & \ldots & 0 \\
0 & -\left(1-\rho_{x}\right) & 1 & -\rho_{x} & \ldots & 0 \\
\vdots & \vdots & \vdots & \vdots & \ddots & \vdots \\
0 & 0 & 0 & \ldots & -1 & 1
\end{array}\right), \\
& L_{v}=\left(\begin{array}{cccccc}
0 & 0 & 0 & 0 & \ldots & 0 \\
-\left(1-\rho_{v}\right) & 1 & -\rho_{v} & 0 & \ldots & 0 \\
0 & -\left(1-\rho_{v}\right) & 1 & -\rho_{v} & \ldots & 0 \\
\vdots & \vdots & \vdots & \vdots & \ddots & \vdots \\
0 & 0 & 0 & \ldots & -1 & 1
\end{array}\right) \text {. }
\end{aligned}
$$

The last vehicle has no follower, so it uses only front spacing and velocity errors. This type of boundary condition is called regular boundary condition [15. The second boundary condition is that the leader is driven independently of the platoon (zeros in the first rows of $L_{x}, L_{v}$ ).

We assume that initially the system in (5) is at standstill and then the leader starts to move with unit velocity:

$$
\begin{gathered}
z_{i}(t)=0 \text { for } i=1, \ldots, N, t<0, \\
x_{0}(t)=0, t<0, x_{0}(t)=t, t \geq 0 .
\end{gathered}
$$

\section{Relation to previous works}

Our paper builds on the results of works [15, 19. Both papers deal with a signal propagation in systems with nearest-neighbor interaction. The vehicle model is a double integrator, i.e., $\ddot{x}_{i}=\epsilon_{i}$ with $\epsilon_{i}$ given in (2), so the papers use nearest-neighbor asymmetric interaction.

The work [19] analyzes a system with a circular topology. We call such system a circular system. The interaction between the leader and the vehicle $N$ is added, so the Laplacians $\hat{L}_{x}, \hat{L}_{v} \in \mathbb{R}^{N+1 \times N+1}$ of a circular graph are

$$
\hat{L}_{x}=\left(\begin{array}{ccccc}
1 & -\rho_{x} & 0 & \ldots & -\left(1-\rho_{x}\right) \\
-\left(1-\rho_{x}\right) & 1 & -\rho_{x} & \ldots & 0 \\
\vdots & \vdots & \vdots & \ddots & \vdots \\
-\rho_{x} & 0 & \ldots & -\left(1-\rho_{x}\right) & 1
\end{array}\right)
$$

$\hat{L}_{v}$ is defined similarly by replacing $\rho_{x}$ by $\rho_{v}$ in 9 .

For such a system, first the conditions for asymptotic stability are found. The most important condition is that $\rho_{x}=0.5$ [19, Prop. 3.5] - there must be a symmetric 
coupling in the position. For stable circular systems it is shown in [19, Thm. 4.8] that an external input or a disturbance causes two signals to propagate in the system in opposite directions and with different velocities. These so-called signal velocities are calculated from the phase velocities [19, Lem. 4.4]. We will use the same ideas in this paper (Sec. 4.2) to describe a stable system in terms of traveling waves.

The paper 15] studies transients in path systems. Its main result is the description of the transient in the path graph using two travelling waves, attenuated at the boundaries [15, Thm. 3.5]. The connection of the circular system from 19 to the path system (which is the one we are really interested in) was conjectured in [15] as follows: the asymptotic instability of the circular system should imply either flock or asymptotic instability of the path system. We now restate flock stability for our system.

Definition 1 (Flock stability, [15]). The system (5) is called flock stable if it is asymptotically stable and if $\max _{t \in \mathbb{R}}\left|z_{0}(t)-z_{N}(t)\right|$ grows sub-exponentially in $N$ for the conditions given in (8).

\subsection{Assumptions for solving}

The solution in 15 is based on two main conjectures relating the path and circular systems. Although we cannot prove them, we use them in the present paper as well. The final justification of both conjectures is in the match of the predicted and simulated values, as shown in Sec. 6 . The first one states that a local behavior of both systems is identical.

Assumption 1. If the path formation (5) is stable and flock stable, then the behavior of a circular system is the same as in the path system for vehicles reasonably far from the boundaries.

This assumption allows to use properties derived for the circular graph (which is much easier to analyze) in the path graph. We note that this treatment implies that the boundary condition for the $N$ th agent in the path graph, if reasonable, does not enter the analysis, and therefore that boundary condition does not affect our conclusions.

A similar assumption has been made by others (see [1, 9, 20]) to simplify the analysis and make the system spatially invariant. In fact, in Solid State Physics this idea is known as periodic boundary conditions and goes back to the beginning of the 20th century (see 21]).

In order to investigate flock stability in the path system using properties of a circular system, we need an additional assumption about relations of the two interconnections.

Assumption 2. If the circular formation is asymptotically unstable, then the path formation is either asymptotically unstable or flock unstable.

The explanation in [15, Def. 3.2] is that the path system has non-normal eigenspaces which makes the bad effects more pronounced. To this explanation we can add

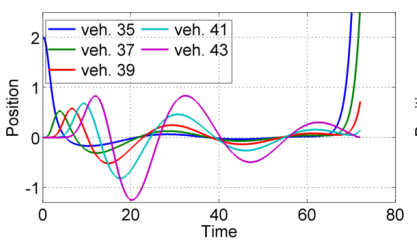

(a) Circular system.

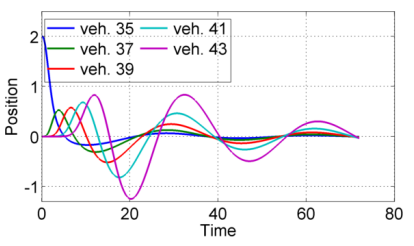

(b) Path system.
Figure 1: Signal propagation in initial-condition response. a) response of an asymptotically unstable circular system, b) response of a flock unstable path system. In both cases $N=70, a=3, g_{x}=2, g_{v}=3, \rho_{v}=\rho_{x}=0.33$.

an additional one based on the travelling wave concept in distributed control [10. Asymptotic instability can be caused by the travelling wave which is amplified as it travels in the formation. The amplification will happen far from boundaries also in the path system. This results either in an asymptotic instability or in a flock instability (if the reflections at the boundaries attenuate the signal sufficiently) of the path system.

Both assumptions are illustrated for our third-order system in Fig. 1 which shows the initial responses $\left(x_{35}(0)=\right.$ 2 and $\left.d_{i, j}=0 \forall i, j\right)$ of the circular and path system. As can be seen, the signal gets amplified as it propagates from one agent to the other. On the other hand, individual agent's response goes to zero, until the amplified travelling wave gets back to the agent after propagating through all other agents (see the sharp growth at time 70 in Fig. 1a). The initial behavior of the path graph and circular systems is the same - both amplify the signal.

\section{Analysis of the circular system}

If $\rho_{x} \neq \rho_{v}$ in the path system in (5), there are two different Laplacians $L_{x}$ and $L_{v}$ which are not simultaneously diagonalizable. This prevents many convenient approaches to guarantee stability such as a synchronization region 22 or LMI-based criterion [23]. This makes the stability and performance analysis of the path system very difficult.

To overcome this limitation, we invoke the Assumptions 1 and 2 to extract some properties of the circular system and apply them in the analysis of the path system. To obtain such properties, we assume in this section that the communication structure is the circular graph with Laplacians $\hat{L}_{x}, \hat{L}_{v}$. These Laplacians are circulant matrices, which are simultaneously diagonalizable. Note that we investigate the circular system only in order to learn something about the path system - the circular system is not of interest by itself.

\subsection{Stability of the circular system}

When we assume the circular interaction topology, the state-space model has a form

$$
\frac{d}{d t}\left(\begin{array}{c}
z \\
\dot{z} \\
\ddot{z}
\end{array}\right)=\hat{M}_{N}\left(\begin{array}{c}
z \\
\dot{z} \\
\ddot{z}
\end{array}\right) \equiv\left(\begin{array}{ccc}
0 & I & 0 \\
0 & 0 & I \\
-g_{x} \hat{L}_{x} & -g_{v} \hat{L}_{v} & -a I
\end{array}\right)\left(\begin{array}{c}
z \\
\dot{z} \\
\ddot{z}
\end{array}\right),
$$


The Laplacians $\hat{L}_{x}, \hat{L}_{v}$ are diagonalizable by the discrete Fourier transform. So let $w_{m}$ be the $m$-th eigenvector of $\hat{L}$, that is the vector whose $j$-th component satisfies

$$
\left(w_{m}\right)_{j}=e^{\imath \phi j} \equiv e^{\imath \frac{2 \pi m}{N+1} j}, \quad j=0,1, \ldots, N,
$$

with $\phi=2 \pi m /(N+1)$ and $\imath=\sqrt{-1}$. By [15], we calculate the eigenvalues $\lambda_{x}$ of $g_{x} L_{x}$ and $\lambda_{v}$ of $g_{v} L_{v}$ as

$$
\begin{aligned}
& \lambda_{x}(\phi)=g_{x}\left[1-\cos \phi+\imath\left(1-2 \rho_{x}\right) \sin \phi\right], \\
& \lambda_{v}(\phi)=g_{v}\left[1-\cos \phi+\imath\left(1-2 \rho_{v}\right) \sin \phi\right] .
\end{aligned}
$$

Let us denote $\beta_{x}=1-2 \rho_{x}, \beta_{v}=1-2 \rho_{v}$. We can expand the eigenvalues $\lambda_{x}$ and $\lambda_{v}$ in the Taylor series

$$
\begin{aligned}
& \lambda_{x}(\phi)=g_{x}\left[\imath \beta_{x} \phi+\frac{1}{2} \phi^{2}-\frac{\imath}{6} \beta_{x} \phi^{3} \ldots\right], \\
& \lambda_{v}(\phi)=g_{v}\left[\imath \beta_{v} \phi+\frac{1}{2} \phi^{2}-\frac{\imath}{6} \beta_{v} \phi^{3} \ldots\right] .
\end{aligned}
$$

We now calculate three eigenvalues $\nu_{m, i}, i=1,2,3$ of $\hat{M}_{N}$ associated with $w_{m}$ for each $m$. The eigenvalue equation is

$$
\left(\begin{array}{ccc}
0 & I & 0 \\
0 & 0 & I \\
-g_{x} \hat{L}_{x} & -g_{v} \hat{L}_{v} & -a I
\end{array}\right)\left(\begin{array}{l}
u_{m, 1} \\
u_{m, 2} \\
u_{m, 3}
\end{array}\right)=\nu_{m, i}\left(\begin{array}{l}
u_{m, 1} \\
u_{m, 2} \\
u_{m, 3}
\end{array}\right)
$$

from which follows that $u_{m, 2}=\nu_{m, i} u_{m, 1}, \quad u_{m, 3}=\nu_{m, i} u_{m, 2}$ and $-g_{x} \hat{L}_{x} u_{m, 1}-g_{v} \hat{L}_{v} \nu_{m, i} u_{m, 1}-a I \nu_{m, i}^{2} u_{m, 1}=\nu_{m, i}^{3} u_{m, 1}$. Since $\hat{L}_{x}$ and $\hat{L}_{v}$ have as an eigenvector $w_{m}$, it follows from the last equality that $u_{m, 1}=w_{m}$. For simplicity of notation we drop the subscripts of $\nu$ except from when ambiguity seems possible. From the last row it is elementary to get a set of $N+1$ eigenvalue equations for $\hat{M}_{N}$ :

$$
\nu^{3}+a \nu^{2}+\lambda_{v}(\phi) \nu+\lambda_{x}(\phi)=0 .
$$

Substituting the expressions for $\lambda_{x}(\phi)$ and $\lambda_{v}(\phi)$, we get

$$
\begin{aligned}
\nu^{3}+a \nu^{2}+ & g_{v}\left[1-\cos \phi+\imath\left(1-2 \rho_{v}\right) \sin \phi\right] \nu \\
& +g_{x}\left[1-\cos \phi+\imath\left(1-2 \rho_{x}\right) \sin \phi\right]=0 .
\end{aligned}
$$

By letting $\phi$ equal 0 or $\pi$ we get real polynomials

$$
\begin{aligned}
\nu^{3}+a \nu^{2} & =0, \\
\nu^{3}+a \nu^{2}+2 g_{v} \nu+2 g_{x} & =0 .
\end{aligned}
$$

The equation (19) implies via Routh-Hurwitz criterion a simple necessary conditions for stability.

Lemma 2. The necessary conditions for the stability of (10) for all $N$ are $a>0, g_{x}>0$ and $g_{v}>0$ and $a>g_{x} / g_{v}$.

An important result is that a symmetric interaction in position is necessary for the stability of the circular system.

Lemma 3. The necessary condition for the stability of (10) for all $N$ is $\rho_{x}=1 / 2$, i.e., $\beta_{x}=0$.
Proof. From (17) for each value of $\phi$ we obtain 3 roots $\nu(\phi)$. Two of the roots in $(18)$ are zero. We will find the behavior of nearby roots (for $|\phi|$ small). Since roots of polynomials are continuous functions of their coefficients, there will be 2 branches of roots with $\nu(\phi)$ tending to zero as $\phi$ tends to zero from above, and another 2 branches with $\nu(\phi)$ tending to zero as $\phi$ tends to zero from below.

Assume that $\beta_{x} \neq 0$. Expand (17) in terms of lowest order (in $\nu^{i} \phi^{j}$ ). We obtain $a \nu^{2}+i \beta_{x} g_{x} \phi=0$. It is easily seen that for all values $|\phi|$ this has two solutions in the right half-plane. It follows that if $|\phi|$ is small enough, (17) must have two solutions in the right half-plane. It follows that we must choose $\beta_{x}=0$, so $\rho_{x}=1 / 2$.

The next theorem guarantees the stability of an arbitrary large system with a circular topology.

Theorem 4. All non-trivial eigenvalues of (10) have negative real part if and only if all of the below hold:

$$
\begin{aligned}
& \text { I. : } \quad a>0 \text { and } g_{x}>0 \text { and } g_{v}>0 \text { and } a>g_{x} / g_{v}, \\
& \text { II. : } \quad \rho_{x}=1 / 2, \\
& \text { III. : } \quad 1-2 \rho_{v} \in\left(-\frac{a g_{v}-g_{x}}{\sqrt{2 g_{v}^{3}}}, \frac{a g_{v}-g_{x}}{\sqrt{2 g_{v}^{3}}}\right) .
\end{aligned}
$$

Proof. Let us call the statement "Circular system $(10)$ is stable" as S. The necessity of conditions I. and II. were proved above. We will use them to prove that given I. and II., then III. is false is equivalent to $\mathrm{S}$ is false.

We know from (18) that $-a$ is a solution of (17) and that it lies in the left half-plane. By continuity of roots of polynomials all the solutions of (17) must lie on a curve $\nu(\phi)$ starting at $-a$. To have unstable roots on the curve $\nu(\phi)$, the curve must cross the imaginary axis for some $\phi \in$ $(0,2 \pi)$. Then there must be purely imaginary solutions $\imath \omega$ ( $\omega$ real) to 17 . Substitute $\imath \omega$ for $\nu$ into 17 to get

$-\imath \omega^{3}-a \omega^{2}+\left[\imath(1-\cos \phi)-\beta_{v} \sin \phi\right] g_{v} \omega+g_{x}(1-\cos \phi)=0$.

The real and imaginary parts of 20 are, respectively:

$$
\begin{aligned}
-a \omega^{2}-g_{v} \beta_{v} \omega \sin \phi+g_{x}(1-\cos \phi) & =0, \\
-\omega\left(\omega^{2}-g_{v}(1-\cos \phi)\right) & =0 .
\end{aligned}
$$

So $S$ is false if both of the last equations hold (i.e., 20 has a solution). The equation (22) holds for $\omega=0$ or $\omega^{2}=g_{v}(1-\cos \phi)$, where $\omega=0$ gives only the 'trivial' eigenvalue (namely $\phi=0$ ). Plugging the other solution $\omega^{2}=g_{v}(1-\cos \phi)$ into 21 gives:

$$
\beta_{v}= \pm \frac{a g_{v}-g_{x}}{\sqrt{2 g_{v}^{3}}} \frac{\sqrt{2(1-\cos \phi)}}{\sin \phi} .
$$

The factor $\frac{\sqrt{2(1-\cos \phi)}}{\sin \phi}$ maps the unit circle onto $[-\infty,-1] \cup$ $[1, \infty]$. So for $\left|\beta_{v}\right| \geq \frac{a g_{v}-g_{x}}{\sqrt{2\left|g_{v}\right|^{3}}}$ there exists $\phi$ for which equation (20) is satisfied, the system then has purely imaginary roots and therefore the system can be unstable. If $\left|\beta_{v}\right|<\frac{a g_{v}-g_{x}}{\sqrt{2\left|g_{v}\right|^{3}}}$, then no imaginary solution exists and whole curve $\nu(\phi)$ lies in the stable half-plane. 


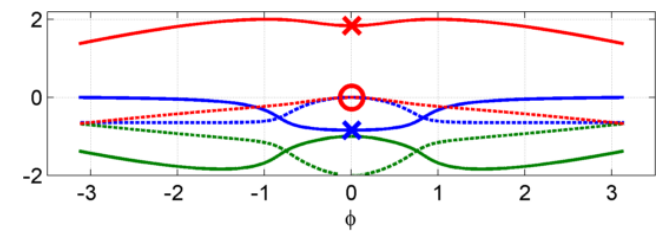

Figure 2: Phase velocities calculated by 24 in vehicles $/ s$ (solid) and the corresponding damping (dashed) as a function of $\phi$. There are three waves for each $\phi$, two with negative velocity (blue and green) and one with positive (red). The phase velocities in green have all high damping, so they do not affect the signal velocity. The signal velocities from $(26)$ are shown by red $\left(c_{+}\right)$and blue $\left(c_{-}\right)$crosses, the corresponding damping by a red circle. $N=500, a=2, g_{x}=6.2, g_{v}=10, \rho_{v}=0.4$.

\subsection{Signal properties}

Similarly to [19], we would like to obtain the signal velocity in our circular system (10). By [19, Lem. 4.4], the phase velocity $c_{m, i}$ and its damping $\alpha_{m, i}$ for a mode associated with a given $\phi=2 \pi m /(N+1)$ can be calculated as

$$
c_{m, i}=-\operatorname{Im}\left(\nu_{m, i}\right) / \phi, \quad \alpha_{m, i}=\operatorname{Re}\left(\nu_{m, i}\right), i=1,2,3 .
$$

We are interested in modes with very low damping, since they travel in the system with slow decay - they give us the signal velocity. Thus, we want to find the eigenvalues $\nu(\phi)$ with small real parts. They are those corresponding to $\phi \rightarrow 0$. To find them, first expand $\nu(\phi)$ as

$$
\nu(\phi)=\imath n_{1} \phi+\frac{1}{2} n_{2} \phi^{2}+\frac{\imath}{6} n_{3} \phi^{3} \ldots,
$$

We substitute the expansions $(13),(14)$ and $(25)$ into $(16)$. Notice that the expansion (25) works because the terms depending on $\phi$ cancel in (17). We collect terms of order $\phi^{2}$, and $\phi^{3}$, etc. The coefficients of these orders must be zero and that will determine $n_{i}$. The first non-trivial equation is the coefficient of $\phi^{2}$. It reads: $\mathcal{O}(2): a n_{1}^{2}+g_{v} \beta_{v} n_{1}-\frac{1}{2} g_{x}=$ 0 . We calculate $n_{1}$ as $n_{1}=\frac{-g_{v} \beta_{v} \pm \sqrt{2 a g_{x}+g_{v}^{2} \beta_{v}^{2}}}{2 a}$.

Since for $\phi$ small by 25 and $24 \operatorname{Im}(\nu(\phi)) \approx n_{1} \phi$, the coefficient $-n_{1}$ determines the signal velocities.

Lemma 5. The signal velocities are given as

$$
c_{ \pm}=\frac{g_{v} \beta_{v} \pm \sqrt{g_{v}^{2} \beta_{v}^{2}+2 a g_{x}}}{2 a},
$$

where $c_{+}>0$ and $c_{-}<0$ (velocity in vehicles/second).

By the stability conditions we mentioned, 26 gives one positive real and one negative real solutions (red and blue crosses in Fig. 22. The wave with the positive velocity $c_{+}$propagates in the direction with growing vehicle index and the wave with $c_{-}$the other way.

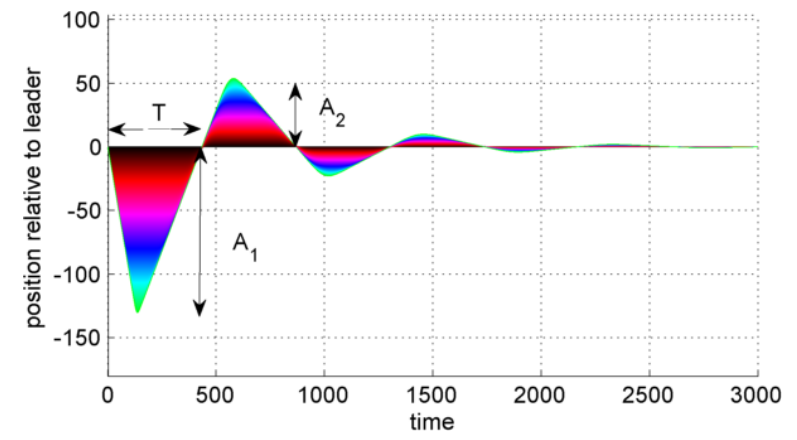

Figure 3: Spacing error to the leader $z_{0}-z_{i}$ with optimized controller (see Sec. 7 and 8). $N=250, a=2, g_{x}=$ $6.2, g_{v}=10$ and $\rho_{v}=0.4$.

\section{Transients in the path system}

We have obtained enough properties of the circular system to derive the transients the original path system (5). The transient we analyze is when the platoon is in steadystate and the leader starts to move with unit velocity (8).

We have Theorem 4 guaranteeing the stability of the circular system which by Assumption 2 allows for flock stability of the path system. The signal velocity in 26 should remain the same in the path system - Assumption 1. The boundary conditions are the same as in [15] - the leader driven independently of the platoon and the agent $N$ having no follower. For stable systems (in both senses), the orbit of the last agent can be characterized by the following quantities (see Fig. 3): half-period $T$ is the smallest $t>0$ such that $z_{N}(t)-z_{0}(t)=0$ and the amplitude $A_{i}$ of the $i$ th oscillation is $A_{i}=\max _{t \in[(i-1) T, i T]}\left|z_{0}-z_{N}\right|$. We can now restate [15, Thm. 3.5] for the system with friction.

Theorem 6. If Assumptions 1 and 2 hold and the path system (5) is asymptotically stable, the parameter values satisfy the conditions in Theorem 4 and as $N$ tends to infinity, the system (5) will behave as a wave equation with boundary conditions. The signal velocities are given by (26). In particular, if from an equilibrium position at rest, the leader starts to move with a unit velocity at $t=0$, then the characteristics of the orbit of $z_{0}(t)-z_{N}(t)$ are:

$$
\begin{aligned}
A_{1} & =\frac{N}{\left|c_{+}\right|}, \\
\left|A_{k+1} / A_{k}\right| & =\left|c_{-}\right| /\left|c_{+}\right|, \\
T & =N\left|\frac{1}{\left|c_{+}\right|}+\frac{1}{\left|c_{-}\right|}\right| .
\end{aligned}
$$

The proof of [15, Thm. 3.5] uses only boundary conditions and wave velocities, hence it remains valid for our case as well. Note that when the leader starts moving, this causes first a wave with velocity $c_{+}$, which then reflects at agent $N$ as a wave with velocity $c_{-}$. Notice that we want $\left|c_{-}\right| /\left|c_{+}\right|$to be less than 1 to avoid exponential growth of the amplitudes. Since $g_{v}$ and $a$ must be positive, we want 
to keep $\beta_{v}>0$, i.e., $\rho_{v}<1 / 2$ and the agent pays more attention to the front velocity error.

\section{Simulation verification}

Fig. 4 numerically validates Theorem 6 by calculating the relative error between the predicted and measured values as a function of $N$. Let $\chi$ be a given quantity of interest in Thm. 6- either $A_{i}, A_{i+1} / A_{i}$ or $T$. Let $\chi_{\text {pred }}$ be the value predicted by (27), 28) or (29), respectively, and $\chi_{\text {meas }}$ be the value measured from the numerical simulations of a finite platoon. The error is calculated as

$$
\vartheta=\log \left(\frac{\chi_{\text {pred }}}{\chi_{\text {meas }}}-1\right),
$$

We can see that the relative error of each predicted parameter decreases exponentially with the increasing number of vehicles in the platoon. This confirms the asymptotic formulas in Theorem 6 and also Assumptions 1 and 2

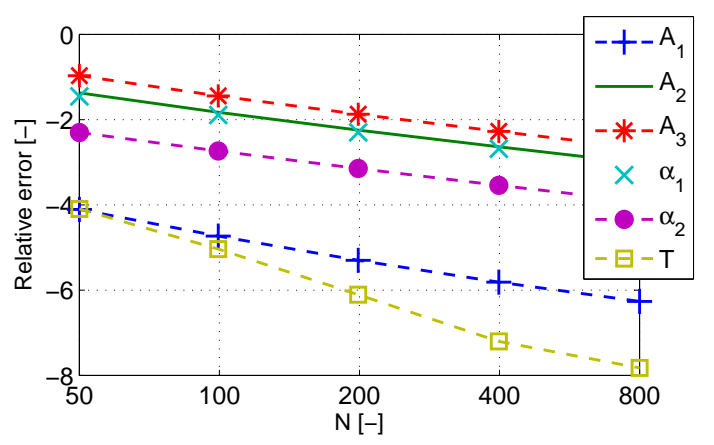

Figure 4: The numerical verification of (27), (28) and 29 . The relative errors are carried out with 30 for $g_{x}=6.2$, $g_{v}=10, a=2, \rho_{x}=0.5$ and $\rho_{v}=0.4$. The $\alpha_{1}$ and $\alpha_{2}$ are the attenuation coefficients from (28) for $A_{2} / A_{1}$ and $A_{3} / A_{2}$, respectively.

The numerical simulations in Fig. 5 show that a platoon with a controller tuned symmetrically (the left panel) has a very long transient. The transient is shortened for the case of the asymmetric controller (the middle panel), however, the overshoot of such a platoon is extremely large, which is a consequence of Lemma 3 - the circular system is unstable, hence the path system is flock unstable. When we set the asymmetry only in the velocity (right), then both the transient and the overshoot are reasonable.

\section{Optimization of system parameters}

The previous section gave us signal velocities and amplitudes of the transient, which depend on the gains $g_{x}$ and $g_{v}$ and the velocity asymmetry $\rho_{v}$. In this section we give an approach how to select these three parameters. We assume that the friction $a$ is given by the vehicle model and cannot be affected by the designer.

We propose the following method for "optimal" (due to asymptotic formulas) gain and asymmetry selection. It is based on minimizing the absolute value of the spacing error of all vehicles in the formation, denoted as $E$, when the leader starts to move from the stand-still. Therefore, the optimization has a form

$$
\min _{g_{x}, g_{v}, \beta_{v}} E=\min \sum_{i=1}^{N} \int_{0}^{\infty}\left|e_{i}(t)\right| d t
$$

where the error is given by $e_{i}(t)=z_{0}(t)-z_{i}(t)$. Clearly, $e_{0}=0$. $E$ is minimized over $g_{x}, g_{v}, \beta_{v}$.

Theorem 6 tells us that the system behaves as a wave equation with boundaries. After a unit change of leader's velocity, first the signal spreads from the leader to vehicle $N$ with velocity $c_{+}$and then it reflects back with velocity $c_{-}$. The graph of the response of the last vehicle in the formation must then be almost triangular, as shown in Fig. 3. The error of the first oscillation for the last vehicle (before $e_{N}$ gets back to zero for the first time) is

$$
E_{N, 1}=\int_{0}^{T}\left|e_{N}(t)\right| d t \approx \frac{1}{2} T A_{1} .
$$

To get the error of the other agents we assume that the maximal value of the error of $i$ th agent is given by $\frac{A}{N} i$ (the peaks are uniformly spaced from 0 to $A_{1}$ with distance $\frac{A_{1}}{N}$ ). Then the shape of the error is almost a trapezoid with one base of length $T$ and the other with $(N-i)\left(\frac{1}{\left|c_{+}\right|}+\frac{1}{\left|c_{-}\right|}\right)=$ $T-\frac{i}{N} T=T\left(1-\frac{i}{N}\right)$. The absolute value of the error of the $i$ th vehicle in the first oscillation is approximately the area of the trapezoid

$$
E_{i, 1}=\int_{0}^{T}\left|e_{i}(t)\right| \approx \frac{A_{1}}{N} i T\left(1-\frac{i}{2 N}\right) .
$$

We have approximated the first oscillations. The errors of the others are calculated in a similar same way, i.e. the period is again $T$ and the amplitude is obtained using (28). The total absolute value of the error of the $i$ th agent is $E_{i}=\int_{0}^{\infty}\left|e_{i}(t)\right| d t$, which is using the trapezoidal approximation given as the sum of areas of all oscillations

$$
\begin{aligned}
E_{i} & =\int_{0}^{\infty}\left|e_{i}(t)\right| d t=\sum_{j=1}^{\infty} E_{i, j} \approx \sum_{j=1}^{\infty} \frac{A_{j}}{N} i T\left(1-\frac{i}{2 N}\right) \\
& =\frac{A_{1}}{N} i T\left(1-\frac{i}{2 N}\right) \frac{1}{1-\frac{\left|c_{-}\right|}{|c+|}} .
\end{aligned}
$$

We used (28) to quantify the amplitude of the $j$ th oscillation and then the sum of geometric series since $\frac{\left|c_{-}\right|}{\left|c_{+}\right|}<1$.

Our criterion (31) captures the sum of $E_{i}$ of all agents. It can be calculated as

$$
\begin{aligned}
E & =\sum_{i=1}^{N} E_{i} \approx \sum_{i=1}^{N} \frac{A_{1}}{N} i T\left(1-\frac{i}{2 N}\right) \frac{1}{1-\frac{\left|c_{-}\right|}{\left|c_{+}\right|}} \\
& =A_{1} \frac{1}{1-\frac{\left|c_{-}\right|}{\left|c_{+}\right|}} T C=J C .
\end{aligned}
$$



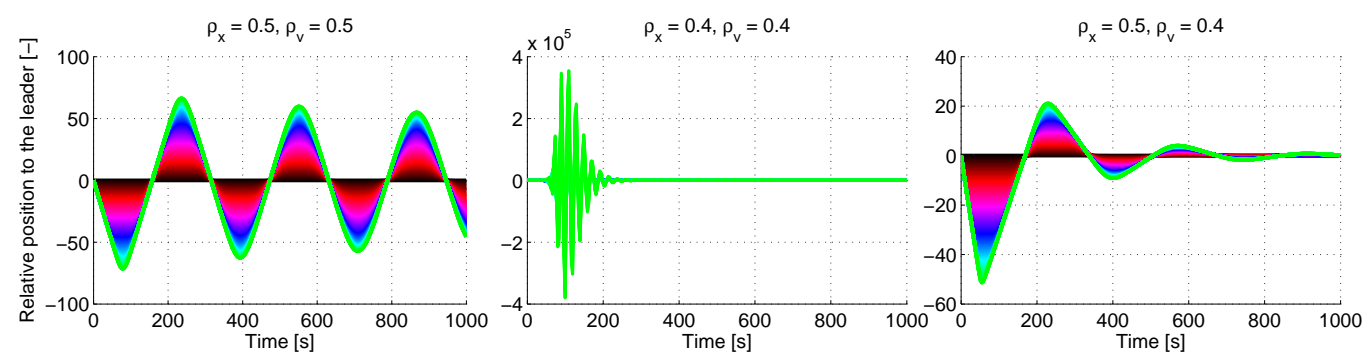

Figure 5: The numerical simulations comparing the responses of three different control strategies for $N=100$, when the leader changes its velocity from 0 to 1 . The figure shows the relative positions to the leader $z_{0}(t)-z_{i}(t)$ of all the vehicles for three different combinations of $\rho_{x}$ and $\rho_{v}$. For all three cases were $g_{x}=6.2, g_{v}=10$ and $a=2$.

with $C=\sum_{i=1}^{N} \frac{i}{N}\left(1-\frac{i}{2 N}\right)$ being a constant which cannot be changed by optimization. Thus, it suffices to minimize $J$. After plugging from 29) and 28, it has a form

$$
J=\frac{A_{1} T}{1-\frac{\left|c_{-}\right|}{\left|c_{+}\right|}}=\left(\frac{\left|c_{-}\right|+\left|c_{+}\right|}{\left|c_{+}\right|\left|c_{-}\right|}\right) \frac{N^{2}}{\left|c_{+}\right|-\left|c_{-}\right|}=\hat{J} N^{2} .
$$

The number of agents is not part of the optimization and does not affect the minimum. Plugging for the signal velocities from $(26)$ we evaluate the sums and products as $\left|c_{-}\right|+$ $\left|c_{+}\right|=\left(\sqrt{g_{v}^{2} \beta_{v}^{2}+2 a g_{x}}\right) / a,\left|c_{-}\right|-\left|c_{+}\right|=\left(g_{v} \beta_{v}\right) / a,\left|c_{-}\right|\left|c_{+}\right|=$ $g_{x} /(2 a)$. With these terms the criterion 36 becomes

$$
\hat{J}=\frac{\sqrt{g_{v}^{2} \beta_{v}^{2}+2 a g_{x}}}{g_{v} \beta_{v}} \frac{2 a}{g_{x}}=\sqrt{\frac{1}{g_{x}^{2}}+\frac{2 a}{g_{v}^{2} \beta_{v}^{2} g_{x}}} 2 a .
$$

Since $a$ is a given constant and the square root is monotonic function, we get the final optimization problem

$$
\min \frac{1}{g_{x}^{2}}+\frac{2 a}{g_{v}^{2} \beta_{v}^{2} g_{x}},
$$

s.t. conditions in Theorem 4,

The final criterion is a function only of the parameters $g_{x}, g_{v}$ and $\beta_{v}$, which the platoon designer can affect.

\subsection{Scaling of the absolute error}

The total error 31 can be written using 35 and 36

$$
E \approx J C=\hat{J} N^{2} \sum_{i=1}^{N} \frac{i}{N}\left(1-\frac{i}{2 N}\right) .
$$

Lemma 7. The error $E$ in (31) scales cubically with $N$ as

$$
E(N) \approx \frac{\hat{J}}{12} N(N+1)(4 N-1) .
$$

Proof. The proof is a simple manipulation of 39 .

$$
\begin{aligned}
E \approx \hat{J} & N^{2} \sum_{i=1}^{N} \frac{i}{N}\left(1-\frac{i}{2 N}\right)=\hat{J} N^{2} \sum_{i=1}^{N} \frac{2 N i-i^{2}}{2 N^{2}} \\
= & \frac{\hat{J}}{2}\left[2 N \sum_{i=1}^{N} i-\sum_{i=1}^{N} i^{2}\right]=\frac{\hat{J}}{2}\left(2 N \frac{N(N+1)}{2}\right. \\
& \left.-\frac{N(N+1)(2 N+1)}{6}\right)=\frac{\hat{J}}{12} N(N+1)(4 N-1) .
\end{aligned}
$$

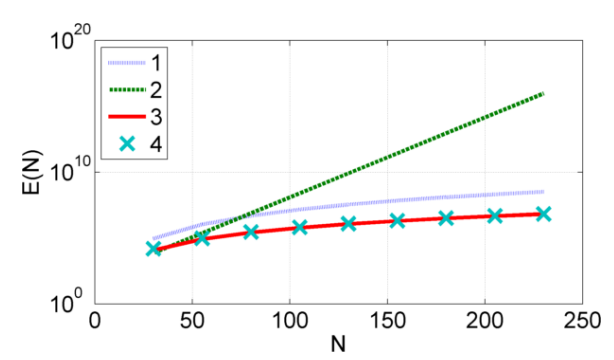

Figure 6: Logarithm of $E(N)$ for different architectures - symmetric with $\rho_{x}=\rho_{v}=0.5$ (line 1), asymmetric with $\rho_{x}=\rho_{v}=0.4$ (line 2) and asymmetric with $\rho_{x}=$ $0.5, \rho_{v}=0.4$ (line 3 ). Line 4 shows estimate using 40). Other parameters were $g_{x}=6.2, g_{v}=10, a=2$.

The scaling of $E$ of different architectures with $N$ calculated from simulations is in Fig. 6. It is clear that the error is the smallest for asymmetry only in velocity and also that the error of asymmetric control with identical asymmetries scales exponentially in $N$, which confirms flock instability $\left(\rho_{x} \neq 0.5\right)$. Also the predicted value matches the calculated one.

\section{Optimization results}

Although both the criterion (38) and the stability constraints are nonconvex, the optimization using function fmincon in Matlab for nonconvex optimization terminated quickly and successfully. The code used for simulations in the whole paper can be obtained at [24].

As follows from (38), the optimization procedure tried to increase the gains $g_{x}$ and $g_{v}$ to decrease the criterion. Therefore we specified upper bounds on $g_{x}, g_{v}$ to limit the controller effort. The optimization was conducted for a given friction $a=2$ and we got the values $g_{x}=6.2, g_{v}=10$ and $\rho_{v}=0.4$. The upper bounds for both gains $g_{x}, g_{v}$ were set to 10. To stay away from the flock stability boundary, we changed the flock stability criterion in Thm. 4 to

$$
\beta_{v}=1-2 \rho_{v} \in\left(-\frac{a g_{v}-g_{x}}{\sqrt{2 g_{v}^{3}}}+\varepsilon, \frac{a g_{v}-g_{x}}{\sqrt{2 g_{v}^{3}}}-\varepsilon\right),
$$

with $\varepsilon=0.1$. The response is shown in Fig. 3 and 5 . 


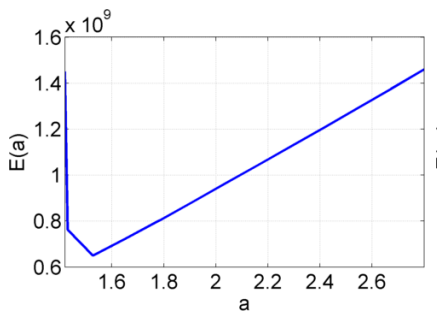

(a) Sens. to change in $a$.

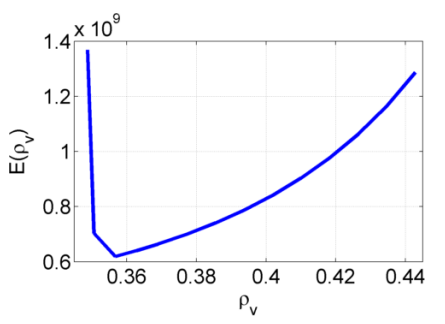

(b) Sens. to change in $\rho_{v}$.
Figure 7: Sensitivities to different parameters. The plots show value of $E$ of (31) calculated from simulations.

\subsection{Robustness evaluation}

The optimization results should be verified to give robust results. The simplest way to achieve robustness is to add some nonzero term $\varepsilon$ to each of the stability criteria in Thm. 4, similarly to (42). Then the system is not allowed to operate on the flock stability boundary.

The most important parameter of the system is the friction $a$. This might change during the operation of the system and also might not be exactly known apriori. Using the values $g_{x}=6.2, g_{v}=10, \rho_{v}=0.4, N=1200$, we simulated the response of the system for friction range $a \in[1.4,2.8]$ and calculated the norm of the error using (31). Fig. 7a shows how the norm changes with friction. We see that the change is approximately linear in friction and the system has a good performance for a wide range of $a$. The sharp growth for low friction caused by a flock instability confirms the Assumption 2. The stability criterion of a circular system in Thm. 4 is violated for $a \leq 1.514$ since $\beta_{v}=1-2 \rho_{v}>\left(\frac{a g_{v}-g_{x}}{\sqrt{2\left|g_{v}\right|^{3}}}\right)$, making the system flock unstable. As $N \rightarrow \infty$, the sharp growth appears exactly at the critical point $a=1.514$.

The Figure $7 \mathrm{~b}$ shows how the error norm changes as a function of $\rho_{v} \in[0.34,0.44]$ with the optimal value $\rho_{v}^{*}=$ 0.37 (other parameters are $g_{x}=8.3, g_{v}=10, a=2, N=$ 1200). These are the values with $\varepsilon=0$ in 42 . It is clear that the value $\rho_{v}^{*}$ is almost the minimum of the function $E\left(\rho_{v}\right)$. The better performance for lower (non-optimal) $\rho_{v} \leq \rho_{v}^{*}$ is due to asymptotic formulas used. When number of vehicles increases, the minimum will get closer to $\rho_{v}^{*}$. Due to the sharp growth for $\rho_{v}<0.36$ we recommend using $\rho_{v}=0.40$ to achieve robustness, as obtained using (42) with $\varepsilon=0.1$. Then we get also $g_{x}=6.2$, as above.

\section{Conclusion}

We investigated the transients in a vehicular platoon with identical vehicles having friction. The asymmetries for a coupling in position and velocity are different. For some steps in the analysis, we considered circular system in order to infer properties of the path system.

To achieve flock stability, the coupling in the position must be symmetric. Then the path system behaves as a wave equation with boundaries, where the travelling waves have different velocities. Using the velocities, we developed an optimization procedure to determine the parameters of the controller. We conclude that the behavior of a platoon with different asymmetries is superior to both symmetric and identically asymmetric platoon.

We believe that the approach and some of the results shown here easily generalize to more complicated systems having two integrators in the open loop.

\section{References}

[1] B. Bamieh, F. Paganini, M. Dahleh, Distributed control of spatially invariant systems, IEEE Trans. Automat. Control 47 (7) (2002) 1091-1107.

[2] F. Lin, M. Fardad, M. R. Jovanović, Optimal control of vehicular formations with nearest neighbor interactions, IEEE Trans. Automat. Control 57 (9) (2012) 2203-2218.

[3] P. Barooah, P. G. P. Mehta, J. J. P. Hespanha, MistuningBased Control Design to Improve Closed-Loop Stability Margin of Vehicular Platoons, IEEE Trans. Automat. Control 54 (9) (2009) 2100-2113.

[4] V. Milanés, S. Shladover, J. Spring, C. Nowakowski, H. Kawazoe, M. Nakamura, Cooperative adaptive cruise control in real traffic situations, IEEE Trans. Intell. Transp. Syst. 15 (1) (2014) 296-305.

[5] J. Ploeg, N. van de Wouw, H. Nijmeijer, Lp String Stability of Cascaded Systems: Application to Vehicle Platooning, IEEE Trans. Control Syst. Technol. 22 (2) (2014) 786-793.

[6] R. H. Middleton, J. H. Braslavsky, String Instability in Classes of Linear Time Invariant Formation Control With Limited Communication Range, IEEE Trans. Automat. Control 55 (7) (2010) 1519-1530.

[7] H. Hao, P. Barooah, On achieving size-independent stability margin of vehicular lattice formations with distributed control, IEEE Trans. Automat. Control 57 (10) (2012) 2688-2694.

[8] J. J. P. Veerman, B. Stošić, A. Olvera, Spatial instabilities and size limitations of flocks, Netw. Heterog. Media 2 (4) (2007) 647-660.

[9] B. Bamieh, M. R. Jovanović, P. Mitra, S. Patterson, Coherence in Large-Scale Networks: Dimension-Dependent Limitations of Local Feedback, IEEE Trans. Automat. Control 57 (9) (2012) 2235-2249.

[10] D. Martinec, I. Herman, Z. Hurák, M. Šebek, Wave-absorbing vehicular platoon controller, Eur. J. Control 20 (2014) 234-248.

[11] H. Hao, P. Barooah, Stability and robustness of large platoons of vehicles with doubleintegrator models and nearest neighbor interaction, Internat. J. Robust Nonlinear Control.

[12] F. M. Tangerman, J. J. P. Veerman, B. D. Stosic, Asymmetric decentralized flocks, IEEE Trans. Automat. Control 57 (11) (2012) 2844-2853.

[13] I. Herman, D. Martinec, Z. Hurák, M. Sebek, Nonzero bound on Fiedler eigenvalue causes exponential growth of H-infinity norm of vehicular platoon, IEEE Trans. Automat. Control 60 (8) (2015) 2248-2253.

[14] H. Hao, H. Yin, Z. Kan, On the robustness of large 1-D network of double integrator agents, in: American Control Conference (ACC), 2012, Montreal, Canada, 2012, pp. 6059-6064.

[15] C. E. Cantos, J. J. P. Veerman, Transients in the Synchronization of Oscillator Arrays (2014) 1-11 arXiv:1308.4919v4

[16] M. Andreasson, D. V. Dimarogonas, H. Sandberg, K. H. Johansson, Distributed control of networked dynamical systems: Static feedback, integral action and consensus, IEEE Trans. Automat. Control 59 (7) (2014) 1750-1764.

[17] P. Wieland, R. Sepulchre, F. Allgöwer, An internal model principle is necessary and sufficient for linear output synchronization, Automatica 47 (5) (2011) 1068-1074.

[18] J. Lunze, Synchronization of Heterogeneous Agents, IEEE Trans. Automat. Control 57 (11) (2012) 2885-2890. 
[19] C. E. Cantos, J. J. P. Veerman, D. K. Hammond, Signal Velocity in Oscillator Arrays, a (2014) 1-18arXiv:1307.7143v3

[20] R. D'Andrea, G. Dullerud, Distributed control design for spatially interconnected systems, IEEE Trans. Automat. Control 48 (9) (2003) 1478-1495.

[21] N. W. Ashcroft, N. D. Mermin, Solid State Physics, Cengage Learning, 1976.

[22] H. Zhang, F. Lewis, A. Das, Optimal design for synchronization of cooperative systems: state feedback, observer and output feedback, IEEE Trans. Automat. Control 56 (8) (2011) 19481952.

[23] P. Massioni, M. Verhaegen, Distributed Control for Identical Dynamically Coupled Systems: A Decomposition Approach, IEEE Trans. Automat. Control 54 (1) (2009) 124-135.

[24] I. Herman, D. Marinec, C. Cantos, J. J. P. Veerman, Code for simulations (2015).URL https://www . mathworks .com/ matlabcentral/fileexchange/52493 